\title{
Las competencias desde la perspectiva informacional: apuntes introductorios a nivel terminológico y conceptual, escenarios e iniciativas
}

\author{
Marlery Sánchez Díaz \\ Máster en Bibliotecología y Ciencias de la Información. \\ Universidad de la Habana. \\ E-mail: infctf@biocen.cu
}

Este trabajo forma parte de la investigación realizada dentro del Programa Doctoral "Documentación e Información Científica"de la Universidad de Granada.

\section{Resumen}

En la actualidad, las organizaciones tienen que enfrentar los cambios en la tecnología, adecuarse a la nueva organización del trabajo y lograr ventajas en el mercado. Para ello, las competencias de los individuos juegan un papel vital, y su gestión se convierte en factor clave para la supervivencia de las mismas. Este enfoque de competencias laborales y/o profesionales, unido a la sociedad de hoy, llamada de la información hace imprescindible las competencias informacionales. Para poder llevar a cabo un estudio aplicado sobre éstas es preciso contar con un acervo teórico-conceptual. A partir de ello, este trabajo tiene como objetivos mostrar, tomando en cuenta primeramente el concepto de competencias, qué se entiende por competencias informacionales a nivel terminológico y conceptual; demostrar, mediante los diferentes escenarios, cómo las competencias informacionales se imponen; y mostrar mediante las distintas iniciativas cómo cada vez resulta más importante su gestión. El trabajo se basa en el análisis documental.

\section{Palabras claves}

Competencias informacionales.

\section{Competences from the informational perspective: introductory notes on terminological and conceptual level, scenarios and initiatives}

\section{Abstract}

At the present time, the organizations have to face the changes in technology, be adapted to the new work organization and take advantages in the market. For that, the competences of the individuals play a vital role, and their administration becomes a key factor for their survival. This focus on labour and/or professional competences, together with today's so called information society, makes information competences essential. In order to carry out a study applied to these competences, it is necessary to have a large theoretical-conceptual collection. From this point of view, taking into consideration first of all the concept of competences, the objective of this paper is to show what is meant by informational competences on the technological and conceptual level and, through different scenarios, how significant the informational competences are; and, finally, to show, through different initiatives, how their management is getting more and more important. This work is based on analysis of documents.

\section{Keywords}

Information competences.

\section{INTRODUCCIÓN}

Ya pasó el tiempo en que los aspectos intangibles de las organizaciones tenían poca importancia. Dentro de éstas, existe hoy una realidad eminente, el factor clave son las personas; es decir, la principal fuente de diferenciación y competitividad es su recurso humano. A los clásicos factores de competitividad, capital financiero y capital tecnológico, se agrega hoy el capital humano. Los conocimientos, capacidades, actitudes, habilidades, aptitudes y experiencias del individuo -es decir, sus competencias; son el centro rector, el principal activo, la fuerza que sostiene a las mismas.

Las competencias, su detección, expresión y desarrollo; resultan esenciales para el éxito de las organizaciones en este mundo en continuo cambio y turbulencia. La supervivencia de éstas están íntimamente ligada a la gestión adecuada de este patrimonio. La gestión por competencias es ante todo un enfoque útil que aporta criterios claros, válidos y efectivos en las difíciles decisiones y actuaciones que afectan a las personas y a los resultados de la organización.

Ahora bien, este enfoque unido a la sociedad de hoy, llamada sociedad de la información ${ }^{1}$, sociedad del conocimiento o sociedad del aprendizaje; donde la información se convierte en un recurso imprescindible y estratégico para la toma de decisiones, la resolución de problemas, el aprendizaje autónomo, el desempeño de todas las funciones organizacionales; hace que dentro del sistema de gestión de competencias no pueden dejar de estar las competencias informacionales.

En cualquier entorno, para lograr el desarrollo social, la información es un elemento clave; por ende se debe prestar significativa atención a que las personas sean capaces de

\footnotetext{
1 Cornella superpone una sociedad de la información en la que la información, simplemente tiene un gran valor, a una sociedad informacional en la que la generación, el proceso y la transmisión de información se convierten en una de las más importantes fuentes de productividad y valor.
} 
interactuar con la información de manera efectiva en toda actividad.

A partir de ello, este trabajo pretende mostrar, mediante análisis documental, qué se entiende por competencias informacionales a nivel terminológico y conceptual; demostrar, mediante los diferentes escenarios, cómo las competencias informacionales se imponen; y mostrar mediante las distintas iniciativas cómo cada vez resulta más importante su gestión.

Para cumplimentar estos objetivos, en un primer momento se aborda como paso previo, aquello de lo que parte la temática, en este caso las competencias -las diferentes definiciones y tipologías. En un segundo momento se delimitan las cuestiones terminológicas relacionadas con el tema. Estos 2 momentos, permiten en uno tercero conceptualizar las competencias informacionales. Con vistas a profundizar en estas ideas, a continuación, se plantean los diferentes escenarios que determinan que en la sociedad actual se hable de competencias informacionales y algunas cuestiones que evidencian la importancia del tema mediante su presencia en las diferentes organizaciones, proyectos y grupos de investigación, las declaraciones y manifiestos, los eventos, publicaciones y recursos electrónicos.

\section{COMPETENCIA(S). DEFINICIONES Y TIPOLOGÍAS.}

Competencia etimológicamente procede del término latino "competere". En el siglo XV el verbo "competir" significó "pelear con", generando sustantivos como competencia, competidor, y el adjetivo, competitivo. (Graminia, 2000) Aquí el término competencia se delimita como la disputa o contienda entre dos o más personas.

"En el siglo XVI en una obra de teatro 'Del rey abajo, ninguno', se decía que nunca ha habido gran hazaña sin haber gran competencia". (Moreno, 1999). El término competencia en este caso aparece como oposición o rivalidad entre dos o más que aspiran a obtener la misma cosa.

Zayas plantea que la introducción del término competencias data de la década de los años 20 en los Estados Unidos en lo referente sobre todo a la capacitación. (Zayas, 2003)

Una vieja definición del Diccionario Larousse de 1930 decía que en los asuntos comerciales e industriales, la competencia es el conjunto de los conocimientos, cualidades, capacidades, y aptitudes que permiten discutir, consultar y decidir sobre lo que concierne al trabajo. Supone conocimientos razonados, ya que se considera que no hay competencia completa si los conocimientos teóricos no son acompañados por las cualidades y la capacidad que permita ejecutar las decisiones que dicha competencia sugiere (Gallart, 1995).
En este caso ya se habla de competencia tomando en cuenta la situación de rivalidad en un mercado ofreciendo o demandando un mismo producto o servicio.

En el contexto empresarial, aparecen autores como Parsons y Atkinson, que comienzan a gestar este concepto. En 1949 Parsons elabora un esquema conceptual que permite estructurar las situaciones sociales, según una serie de variables, una de ellas consistía en valorar a una persona por la obtención de resultados concretos en vez de hacerlo por una serie de cualidades que le son atribuidas de una forma más o menos arbitraria. En 1958 Atkinson logra demostrar de forma estadística la utilidad de dinero como un incentivo concreto que mejora la producción siempre que el mismo estuviese vinculado a resultados. (Cuesta, 2001)

Hooghiemstra plantea que en 1966 y 1968 autoridades como Ghiselli y Mischel respectivamente hicieron afirmaciones en cuanto a la relación de los rasgos comprobables de personalidad y desempeño del trabajo. (Hooghiemstra, 1996)

McClelland realiza una serie de estudios sobre los aspectos motivacionales en relación con el éxito profesional. Es en 1973 cuando manifiesta que por si solos, los expedientes académicos y los test de inteligencia no eran capaces de predecir con fiabilidad la adecuación de las personas a los puestos de trabajo y por tanto a la consecución del éxito profesional y como consecuencia de tales conclusiones acuñó el término "competency/competencies". (McClelland, 1973).

McClelland logró confeccionar un marco de características que diferenciaban los distintos niveles de rendimiento de los trabajadores a partir de una serie de entrevistas y observaciones. La forma en que describió tales factores se centró más en las características y comportamientos de las personas que desempeñaban los empleos que en las tradicionales descripciones de tareas y atributos de los puestos de trabajo (Spencer, 1994).

En la literatura aparecen múltiples y variadas conceptualizaciones de competencias, como consecuencia de los distintos orígenes profesionales de los autores, las diferentes interpretaciones, las distintas maneras de entender el término. Así lo reconoce Cuesta cuando plantea que "... no hay consenso acerca de la concepción de gestión de competencias ni sobre la propia acepción de competencias. En eso influyen su condición de intangible, la interdisciplinariedad demandada por su enfoque y lo reciente de su incorporación al campo de la gestión empresarial”. (Cuesta, 2002).

En palabras de Le Boterf "la dificultad de definirlo crece con la necesidad de utilizarlo”. (Le Boterf, 1997).

Al no existir una definición unívoca aceptada universalmente de competencias y que el concepto aún está en etapa de 
discusión y elaboración, se plantean diversas cuestiones a partir de algunas definiciones localizadas en la literatura (McClelland, 1973; Boyatzis, 1982; Masterpasqua, 1991; Spencer, 1993; Wordruffe, 1993; Bunk, 1994; Feliú, 1994; Hamel, 1994; Moore, 1994; Reis, 1994; Tremblay, 1994; INEM, 1995; AFNOR, 1996; Ansorena, 1996; Gonczi, 1996; Irigoin, 1996; Mertens, 1996; Gallart, 1997; Cowling, 1997; Ducci, 1997; Levy-Leboyer, 1997; Le Boterf, 1997; Agudelo, 1998; IHCD, 1998; Kochansky, 1998; Lasida, 1998; Muñoz, 1998; Novick, 1998; Tejada, 1999; Zarifian, 1999; Consejo Federal de Cultura y Educación de Argentina, 2000; Ibarra, 2000; Norton, 2000; Ruiz, 2000; Vargas, 2000; Cruz, 2001; ISPETP, 2002; Ravitsky, 2002; Pissinati, 2002; González, 2004; Ponjuán, 2004).

Algunos autores hablan de la diferencia entre competencia en singular y competencias en plural, entre ellos Mertens, Zarifian, Vargas.

Para esta autora competencia (en singular) es cada vez más un concepto para señalar la capacidad real de alcanzar objetivos (laborales u organizacionales) mediante la movilización de ciertos recursos (competencias en plural).

En los conceptos analizados, los términos que prevalecen son:

a) los conocimientos (Masterpasqua, Bunk, Tremblay, Le Boterf, Irigoin, Cowling, Kochansky, Muñoz, Tejada, Consejo Federal de Cultura y Educación de Argentina, Ibarra, Norton, Cruz, Ravitsky, ISPETP, Pissinati, Ponjuán);

b) las habilidades (Masterpasqua, Spencer, Feliú, Tremblay, Le Boterf, Ansorena, Gonczi, Irigoin, IHCD, Kochansky, Muñoz, Tejada, Consejo Federal de Cultura y Educación de Argentina, Ibarra, Norton, Cruz, Ravitsky, ISPETP, Pissinati, Ponjuán);

c) las actitudes (Wordruffe, Feliú, Ansorena, Le Boterf, Cowling).

Entonces, se puede decir, en un principio, que las competencias son el conjunto de estos recursos...

También, aparecen de manera explícita, aunque en menos medida: las capacidades, las destrezas, las aptitudes, los rasgos de la personalidad, los atributos y la experiencia. En algunos se habla de la motivación, los valores y los saberes.

O sea, es importante la combinación y mezcla adecuada de estos recursos: saber (conjunto de conocimientos y experiencias, dependen totalmente del portador del conocimiento); saber conocer (dependen totalmente del portador del conocimiento); saber hacer (habilidades técnicas, sociales y cognitivas, dependen totalmente del portador del conocimiento); saber ser (aptitudes, es el punto donde se unen los intereses individuales con los intereses organizacionales); saber ser con los demás (es el punto donde se unen los intereses individuales con los intereses organizacionales); saber estar (aspectos motivacionales de carácter internos y externos, es el punto donde se unen los intereses individuales con los intereses organizacionales); querer hacer (actitud de implicación); poder hacer (desde el punto de vista individual y situacional es totalmente responsabilidad de la organización y más bien se encuentra relacionado con el sistema estructural que la compone y se relaciona en forma de conocimientos encapsulados).

A partir de esto, se puede decir que las competencias tienen un enfoque holístico, sinérgico y sistémico pues ellas incluyen no sólo conocimientos y habilidades para hacer el trabajo (saber hacer), sino también actitudes, valores y rasgos personales vinculados al buen desempeño en el mismo (querer hacer).

El plantearse las competencias solamente a un componente, niega el enfoque dialéctico de este proceso que como sistema están estrechamente intervinculados y estructurados para el desempeño de una actividad.

La mayoría de las definiciones estudiadas direccionan hacia o desde el mundo del trabajo -Boyatzis, Punk, Moore, Irigoin, Agudelo, Ibarra, Cruz, Ravitsky, ISPETP, González.

Es válido enfatizar aquí, que la definición de las competencias exige acuerdo y colaboración entre el mundo de la educación y el mundo del trabajo, pues éstas se adquieren en trayectorias que implican una combinación de educación formal, aprendizaje en el trabajo (no formal) y eventualmente, educación informal.

Algunos autores centran su definición en comportamientos observables, tal es el de Ponjuán; sin embargo, otros como Boyatzis y Spencer, en rasgos subyacentes de la persona.

En este sentido, esta autora es del criterio que las competencias, para considerarse como tal, deben ser demostrables y por ende observables.

-Determinadas definiciones explicitan las características -Spencer, Kochansky; las calificaciones; las capacidades -Moore, Reis, AFNOR, Muñoz; y los roles -Tejada.

Tomando en cuenta estas cuestiones, es preciso diferenciar las competencias de:

a) Las características de las personas. Estas características se convierten en competencias cuando se demuestra que resultan útiles para una actividad determinada. 
b) Las calificaciones. "Por calificación se entiende el conjunto de conocimientos y habilidades que los individuos adquieren durante los procesos de educación formal y la competencia se refiere a ciertos aspectos de este acervo de conocimientos y habilidades, los necesarios para llegar a ciertos resultados exigidos en una circunstancia determinada y a la capacidad real para lograr un objetivo en un contexto dado." (Mertens, 2002).

c) El ser capaz. Las competencias son inseparables de la acción, se ponen de manifiesto cuando se ejecuta una tarea, actividad o un trabajo, serán apreciadas cuando hayan evidencias o resultados. Tiene que estar la demostración, no se identifican con realizaciones aisladas y puntuales, sino con desempeños que expresan el dominio de una práctica.

d) Los roles y las funciones. Las competencias son el conjunto de conocimientos, habilidades y actitudes que son críticos para el desempeño de un rol (tarea o actividad de una profesión); o función (actividades o tareas que ejecutan las personas que desempeñan un rol específico).

A partir del estudio efectuado se puede concretar que:

Las competencias consisten en la puesta en acción, en práctica, en juego; en la movilización de forma combinada, integrada; en un contexto determinado, de todos los recursos (habilidades, conocimientos y actitudes) para la resolución de problemas y situaciones diversas, de forma exitosa.

Tal y como cada autor emite su definición de competencias, también tipifican a las mismas. Estas tipologías son también muy controvertidas e indistintas, aunque de alguna manera todas tratan de expresar lo mismo, o sea, los autores denominan la idea central con nombres distintos.

A partir de esta situación, se puede plantear, a partir de los diferentes autores (Cejas, s/f ; Bunk, 1994; Gallart, 1995; Mertens, 1996; Zarifian, 1996; Levy-Leboyer, 1997; Le Boterf, 1997; Cardona, 1999; Tejada, 1999; Cruz, 2001; Zayas, 2003; Vargas, 2004) que se tiende a agrupar las competencias en:

Generales - genéricas - transversales - horizontales.

Específicas - particulares - de puestos - técnicas verticales.

Las primeras son las necesarias para todas las profesiones en mayor o menor medida, son transferibles a una gran variedad de funciones y tareas, no son exclusivas a ninguna especialidad profesional, permitirán afrontar las competencias específicas del área de conocimiento propio; y las segundas son las propias de cada perfil profesional con su tronco y sus especializaciones.
Hay contradicciones entre los autores pues unos hablan de claves refiriéndose a las que han de ser adquiridas en la formación obligatoria. Para esta autora éstas son básicas o genéricas; y las claves son las centrales y/o estratégicas para la organización.

Estos tipos de competencias no son las únicas, también se habla de directivas, intelectuales, evaluativas, instrumentales, blandas y duras, individuales (personales) y organizacionales (sistémicas o colectivas), desarrollables y poseídas, formales (reglamentadas) e informales (no reglamentada, aprendizajes previos y por experiencia laboral) simples y complejas.

Muchos autores tratan la competencia social como aquella que permitirá la interacción social.

Algunos autores establecen la diferencia entre competencia laboral y profesional. Aquí es importante precisar que, lo laboral implica todo lo relacionado con el mundo del trabajo, ya sea profesión u oficio.

\section{CUESTIONES TERMINOLÓGICAS RELACIONADAS CON LAS COMPETENCIAS INFORMACIONALES}

El tema de las competencias informacionales, los autores lo abordan utilizando diferentes terminologías: competencias en información (Angulo, 2007); competencias informativas (Cortés, 2003; Moreira, 2006; Hernández, 2007); competencias informacionales (Ortoll, 2003); competencias relacionadas con la gestión de información ${ }^{2}$ (Lau, 2007); competencias en el manejo de la información (Muntada, 2003; Pinto, 2006); competencias para el manejo y uso de la información. (Córdoba, 2007); competencias en alfabetización informacional (Pasadas, 2007).

Según Lau, el adjetivo "informativo", denota "necesidad de informar" de manera pura, sin mayor atención a la aplicabilidad; mientras que "informacional", refleja un sentido de "aplicabilidad a algo", lo cual lo hace más apropiado desde el punto de vista semántico. Este autor considera que cualquiera de los dos adjetivos puede considerarse correcto. (Lau, 2007).

Para Hernández deberían ser informativas, puesto que el término informacionales no existe en español. (Hernández, 2007).

Information competente (Curzon, 1995), information competency (Dodge, 2003; Watson, 2003) , information competencies

2 Proceso mediante el cual se obtienen, despliegan o utilizan recursos básicos (económicos, físicos, humanos, materiales) para manejar información dentro y para la sociedad a la que sirve. Tiene como elemento básico la gestión del ciclo de vida de este recurso y ocurre en cualquier organización. (PONJUÁN, G. Gestión de Información: Dimensiones e Implementación para el éxito Organizacional. NUEVO PARHADIGMA 2004 Rosario Argentina. 216p.) 
(Weiner, 2001), information competence skills; son los términos empleados por la literatura de habla inglesa. En francés compétences informationnelles (Chevillotte, 2005).

Es importante apuntar que la terminología suscita controversias, pues varía según los contextos, los sectores profesionales; entre otras cuestiones. Es por eso que muy relacionado con las competencias informacionales aparece en la literatura la alfabetización informacional.

El término alfabetización ha entrañado distintos significados a través del tiempo, las circunstancias y los distintos autores: capacidad de leer y escribir, tener alguna habilidad o competencia (Snavely, 1997; Gómez, 2002) y el aprendizaje.

Para determinados autores la alfabetización adquiere significados y dimensiones en función de la necesidad de competencias de cada época y lugar; por tanto, éstos no identifican alfabetización con su sentido literal de adquisición de la lectoescritura; sino de una manera global. Y consideran que poner un segundo nombre es empobrecer el sentido de ésta. Cornella expone que en la sociedad del conocimiento, el concepto de alfabetización adopta un nuevo significado. (Cornella, 1999).

Para otros existen diferentes alfabetizaciones como son: digital, informacional, audiovisual, multimedia, entre muchas otras. La comunidad profesional relacionada con las instituciones de información se inclina por estos tipos de alfabetización, es por eso que se habla de múltiples caras de la alfabetización o alfabetizaciones múltiples. También de alfabetizaciones basadas en destrezas.

Al revisar las diferentes fuentes de información se puede observar que la alfabetización informacional se relaciona con el conjunto de conocimientos, destrezas, capacidades, saberes, habilidades, conductas, aptitudes; es decir competencias relacionadas con la información (Taylor, 1986; Tuckett, 1989; Bruce, 1997; Gómez, 2000; Gómez, 2002; Angulo, 2003; Declaración de Praga, 2003; Byrne, 2005; Declaración de Alejandría, 2005).

Todo esto trae consigo que la expresión "information literacy" (infoliteracy) sea traducida por unos como es el caso de Gómez como alfabetización informacional; Enebral habla de destreza, suficiencia o excelencia (Enebral, 2006); mientras que otros eligen competencia informacional como por ejemplo Snavely. (Snavely, 1997).

Gomez propone la traducción de information literacy por alfabetización informacional desde 1998 frente a otras traducciones. Según él,

"es correcta gramaticalmente pues información admite la derivación informacional, que se usa también con otros términos: cultura, economía, pedagogía... informacional". (Gómez, 2005) y es la que cree que más se ajusta a su significado en el contexto del que parte.

Hay autores que exponen la Alfabetización Informacional como competencia transversal clave en la sociedad del conocimiento. (Pinto, 2006); Information Literacy competencies (Manuel, 2004) e Information Literacy competency.

Benito plantea cómo, está adquiriendo gran relevancia, el desarrollo de actividades para mejorar las competencias en el manejo de información, que se integra dentro de un nuevo paradigma formativo denominado alfabetización informacional. (Benito, 2007a).

Vale aclarar que unos utilizan para la alfabetización informacional acrónimos como ALFIN (Pasadas, 2003) y otros INFOLIT (Benito, 2000).

La alfabetización informacional se utiliza para denominar los servicios diseñados para facilitar la adquisición de las capacidades informacionales; desde el punto de vista teórico o de la investigación, aparece como un área disciplinar cuyo objeto es el desarrollo de normas, modelos pedagógicos, criterios de evaluación, estrategias políticas para la mejora de las competencias informacionales de los ciudadanos... (Gómez, 2005).

Existen autores como por ejemplo Ortoll, que consideran las competencias informacionales más allá de la alfabetización informacional. Ella expone que las competencias informacionales incluyen además de aprender a utilizar la información con independencia de la localización de la fuente, el relacionarse de forma efectiva con el mundo de la información y compartir conocimiento en un contexto determinado. La alfabetización informacional va dirigida a todos los miembros de la comunidad y puede ser impartida fuera de la ubicación de la institución de información; sin embargo las competencias informacionales se dirigen a los profesionales y futuros profesionales y es impartida en los distintos escenarios de formación de los profesionales. La alfabetización en información es impartida en colaboración con otros profesionales como materia central y las competencias informacionales son impartidas por los distintos agentes responsables de la formación de competencias profesionales (Ortoll, 2003).

En este sentido se realiza una búsqueda en la base de datos LISA $^{3}$ y se puede decir que a partir del 2000 la mayor cantidad de trabajos responden al término "information literacy" con 106 registros, siguiéndole el "user training" como tema significativo con 96 trabajos. Muy pocos registros responden a "attitudes" "skills" "competences" relacionados con la información. Al seguir

3 Library and Information Science Abstracts (1969--) 
el estudio que realiza Bawden (Bawden, 2002), que abarca 1980 1998; si se compara la producción científica de los años 90, con lo que va de década se observa un ascenso considerable. Esto también es corroborado al realizar otra búsqueda, pero en el Sciences Citation Index - SCI, donde se pudo observar que a partir del año 2000 la producción de trabajos relacionados con el tema se duplicó. En los artículos indexados, también se observó la presencia de la alfabetización computacional y medial.

\section{DEFINICIÓN DE LAS COMPETENCIAS INFORMACIONALES}

A partir de algunas de las definiciones de competencia(s) informacional(es) localizadas se pueden plantear diferentes cuestiones (Zurkowski, 1974; Burchinal, 1976; Hamelink, 1976; Owens, 1976; Garfield, 1979; Taylor, 1979; Horton, 1983; Fjallbrant, 1984; Breivik, 1985; Fatzer, 1987; ALA, 1989; Kuhlthau, 1989; Olsen, 1989; Tuckett, 1989; Rader, 1991; Bjoner, 1991; Pinto, 1991; Lenox, 1992; Behrens, 1994; Candy,1994; McClure, 1994; Rogers, 1994; Curzon, 1995; Shapiro, 1996; Barry, 1997; Goad, 1998; Goetsch, 1998; Hernández, 1998; Mackenzie, 1998; Morales, 1998; Spitzer, 1998; Hepworth, 2000; Tedesco, 2000; Cornella, 2001; Brose, 2002; Muntada, 2003; Virkus, 2003; Webber, 2003; Montero, 2004; Byrne, 2005; Gómez, 2005; Brindley, 2006; Candy, 2006; Benito, 2007b; Dolan, 2007; Lau, 2007; Sonntag, 2007):

Algunos autores utilizan el singular como Kuhlthau, Shapiro y Goetsch; otros el plural como Zurkowski y Gómez.

En muchas ocasiones las competencias informacionales se ven como sinómino de las aptitudes informativas y de las habilidades informacionales. Es preciso plantear que en muchas traducciones del inglés al español, se ha utilizado el término aptitudes cuando aparece "competency". Sin embargo, las competencias informacionales no son más que el conjunto de estos recursos, integrados y movilizados...

El comportamiento de estos recursos se presencia de forma distinta en las definiciones. La mayor cantidad de autores consideran que las competencias informacionales están constituidas por habilidades, a partir de los diferentes procesos -Zurkowski, Burchinal, Owens, Breivik, Fatzer, ALA, Olsen, Rader, Lenox, Beherens, Candy, Curzon, Goetsch, Spitzer, Hernández, Mackenzie, Hepworth, Cornella, Montero, Byrne, Lau; otros incluyen además los elementos cognitivos -Zurkowski, Horton, Breivik, ALA, Bjoner, Candy, Curzon, Shapiro, Goetstch, Spitzer, Hernández, Cornella; y algunos incorporan la actitud-Breivik, Bjoner, Hernández, Byrne.

Este concepto ha sido enfocado hacia los diferentes ámbitos:

a) el académico -Breivik, Bjoner, Lenox, Curzon, Goesch; b) el laboral -Zurkowski, Burchinal, Owens, Cornella y

c) de manera general -Taylor, Horton, ALA, Rader, Hernández, Montero, Webber. La mayoría lo enfocan en esta última forma.

Parece haber 2 tendencias: la que plantea la competencia informacional en un sentido más amplio y global en la que sea aplicable en cualquier situación e independiente de la disciplina o campo de conocimiento implicados en la misma y la que considera que la competencia informacional se aplica a un contenido específico -Candy y Hepworth.

Las competencias informacionales entran dentro de las competencias transversales y claves; pues son necesarias a todas las disciplinas, a todos los entornos de aprendizaje y a todos los niveles educativos. No importa el tipo de profesional o el tipo de actividad pues son imprescindibles en cualquier práctica laboral, sea en procesos de negocios, gerenciales y/o técnicos.

Pero es importante plantear que como materia central forman parte de las competencias transversales para el desarrollo de una actividad profesional, pero tienen especificaciones para las diferentes disciplinas.

La mayoría de las definiciones explicitan la solución de problemas de corte informacional o de otra naturaleza-Zurkowski, Burchinal, Owens, Garfield, Taylor, Horton, Brevik, ALA, Olsen, Rader, Bjoner, Lenox, Goetsch, Spitzer, Cornella.

Otras incluyen el aprendizaje a lo largo de la vida -ALA, Lenox, Candy, Montero, Breivik, Dolan.

El concepto de competencia informacional ha ido evolucionando a lo largo de los años, esto se argumenta con el siguiente análisis. El término, aparece en Zurkowski en 1974; aunque es en los años 90 cuando cobra fuerza. Este autor lo enmarca en el ambiente laboral. Burchinal es uno de los primeros autores que en 1976 plantea la resolución de problemas y amplia la concepción en relación con Zurkowski. Hamelink en 1976 va más allá de la búsqueda de información, pues plantea ya el formarse criterios independientes. Owens también en 1976 habla de individuos informacionalmente incompetentes y de la necesidad de tener competencias informacionales para la toma de decisiones adecuada (aquí la toma de decisiones va más allá de la naturaleza informacional). Garfield en 1979 plantea las habilidades técnicas. Así también en ese mismo año Taylor introduce que el proceso de informarse es continuo y que por tanto es tan importante como el proceso de búsqueda de información, que es ocasional. Y en 1985 Breivik habla de un conjunto integrado de habilidades, conocimientos, herramientas y recursos a partir de las actitudes. En 1989 Tuckett considera 3 niveles: destrezas de información sencillas, destrezas en información compuestas y destrezas de información integradas. Rader en 1991 es del criterio que la competencia en información va más allá de la biblioteca, y de forma tanto local como global; Bjoner introduce 
la motivación y Pinto la aptitud. McClure en 1994 plantea la consideración de la información en cualquier formato y la coloca en la intersección de la alfabetización tradicional, informática, en medios y en redes. Mientras que Curzon en 1995 expone que es la fusión o la integración de la formación de usuarios de bibliotecas, la competencia informática, la cultura de los medios de comunicación, competencia tecnológica. Shapiro en 1996 considera que se debe ver como una parte esencial del contexto mental de un individuo educado en la era de la información. Morales en 1998 en su concepto incluye los valores y direcciona la competencia informacional con vistas a hacer de los ciudadanos personas que sepan actuar de forma autónoma y estratégica, capaces de enfrentarse a situaciones y contextos cambiantes, preparados tanto para aprovechar los propios recursos mentales, autorregular el aprendizaje, actuando de forma participativa y solidaria. Hepworth en el 2000 expone 4 áreas de aprendizaje: aprender a usar las herramientas de información, aprender procesos intelectuales asociados a la creación de conocimiento y a la gestión de información, aprender a comunicar y aprender las normas intelectuales asociadas a un área de conocimiento. Cornella en el 2001 enfatiza la competencia en información para las actividades diarias, en el hogar, en el trabajo, y en los actos sociales. Webber en el 2003 habla de conducta, de los distintos medios o canales y de la ética.

No en todos los autores aparecen los mismos elementos. Por ejemplo en la definición de Zurkowski solamente está presente la competencia relacionada con el uso de la información; mientras que la de la American Library Association (ALA) incluye la de la localización y evaluación de los recursos de información. Vale destacar que las competencias informacionales también abarcan la comprensión, utilización y comunicación de la información.

Algunas definiciones, como por ejemplo la de Candy, Curzon y Brose; explicitan que las competencias informacionales incluyen todo tipo de información (gráfica, multimedia, textual); fuentes de información (documentales, no documentales), formatos (impresos, electrónicos), soportes, canales, ubicación, organización de contenidos (secuencial, hipertextual).

Se discute de manera confusa y controvertida, la relación entre las competencias informacionales con las habilidades para el uso de la biblioteca y sus servicios y con las habilidades en la búsqueda de información -Fjallbrant, Rogers, Barry, Goad, Muntada, Gómez, Virkus. Hay autores que plantean que se ha adoptado el término competencia informacional para la tradicional formación de usuarios e instrucción bibliográfica; otros son de la opinión que las habilidades implicadas en la formación de usuarios y la instrucción bibliográfica constituyen una parte importante para el logro de las competencias informacionales.

-Para muchos autores las competencias informáticas son necesarias para el logro de las competencias informacionales -Horton, Taylor, Pinto, Morales, Cornella, Candy. Es preciso enfatizar que el concepto de competencias informacionales va más allá de las competencias informáticas.

Existe controversia también en cuanto a si las competencias informacionales incluyen las competencias mediáticas, comunicacionales, visuales, entre otras; o si son la fusión de todas -Kuhlthau, Benito.

El pensamiento crítico forma parte de muchas de las definiciones estudiadas -Curzon, Goetsch.

Es evidente que el logro de este tipo de competencias requiere actitud hacia el trabajo en grupos y la confianza hacia los demás.

Tomando como base el enfoque de competencias - en cuanto al concepto y tipos de competencias -, las delimitaciones terminológicas y el análisis de las diferentes definiciones relacionadas con las competencias informacionales se puede plantear que:

Las competencias informacionales, consisten en poner en acción, en práctica, en juego, en movilización; de forma combinada, mezclada o integrada; en un contexto y con un contenido determinado (transferibles), todos los recursos (habilidades, conocimientos, actitudes); para solucionar con éxito problemas y aprender a aprender, a partir de la interacción efectiva con la información; sin delimitación de tipo, formato y soporte. Son necesarias en cualquier ámbito e incluyen otras competencias como las tecnológicas, las bibliotecarias, las bibliográficas, el pensamiento crítico y las sociales.

\section{ESCENARIOS E INICIATIVAS RELACIONADAS CON LAS COMPETENCIAS DESDE LA PERSPECTIVA INFORMACIONAL}

Existen diferentes escenarios que determinan que en la sociedad actual se hable de competencias informacionales, dentro de los que se encuentran:

Cada uno de los miembros de la sociedad necesita buscar por sus propios medios la información que le requiere su trabajo, su estudio y hasta su vida cotidiana (Angulo, 2003); requieren competencias para lograr acceder y utilizar esa información...

Existe una sobreabundancia de información, donde el manejo de ésta resulta cada vez más difícil y conflictivo y provoca saturación de información. Cornella habla entonces de infoxicación (Cornella, 2001) y Wurman de ansiedad informativa (Wurman, 1989). Por ende la mayor cantidad de información existente exige comprensión, análisis y evaluación.

"Es imposible diseñar programas educativos donde se transmitan a los estudiantes todos los contenidos relevantes 
sobre un determinado tema, en su lugar los estudiantes deben desarrollar las competencias que les permitan ser autosuficientes en el acceso y aprovechamiento de la información" (Cortés, 2003) y lograr el autoaprendizaje. (ANUIES, 2000).

Se hace necesaria la transición de un modelo de aprendizaje basado en canales de información establecidos, a otro, en el que existe un número y una variedad mucho mayor de datos caóticos a los que se accede a través de unos canales más fluidos y variados.

El tener acceso a la misma información que los demás, posee cada vez menos valor; y el descubrir nuevas fuentes y sintetizar su información con la ya disponible, adquiere una enorme y creciente importancia.

La toma efectiva de decisiones necesita ser construida sobre un acceso oportuno a la información adecuada y la capacidad para procesar la información disponible y ajustarse a las demandas de la decisión.

La rápida explosión de recursos de información y el desarrollo tecnológico hace que las personas tengan que aprender a elegir recursos, encontrar y seleccionar información, hacer el mejor uso de ella y moverse de forma efectiva en el contexto informacional.

En un mundo cada vez más global y cambiante, será esencial el aprendizaje de estrategias para resolver problemas, tomar decisiones, desarrollar nuevas habilidades para adaptarse a las nuevas demandas laborales y tecnológicas...y aprender a lo largo de la vida. Se hace imprescindible la necesidad de actualización y ampliación constante de los conocimientos.

A partir de estos escenarios, es eminente, la exigencia indiscutible de lograr las competencias informacionales de los individuos en grado óptimo y a un nivel de excelencia.

En función de todos estos escenarios y de la necesidad de gestionar las competencias informacionales; se discute el rol que juega el bibliotecario, su labor como docente y la necesidad de nuevas competencias para este profesional.

La Organización Internacional del Trabajo (OIT), la UNESCO y numerosas naciones han reconocido la "infoalfabetización" como factor clave de empleabilidad, de la capacidad individual de mantener una actividad laboral satisfactoria en lo profesional, económico y personal, como también de un elemento esencial en la continuidad de estudios de un profesional. (Pasadas, 2004)

Desde la década del 90 existen diferentes iniciativas de los gobiernos e instituciones educativas para fomentar la competencia informacional.
Los siguientes enunciados demuestran que el tema de las competencias informacionales, se ha ido imponiendo:

-El Reporte de la Secretary's Commission on Achieving Necessary Skills identifica las competencias informacionales dentro de las 5 categorías de competencias transversales a cualquier tipo de trabajo. (SCANS, 1991)

-La Organisation for Economic Co-operation and Development plantea que la creciente convicción de la importancia de la información y el conocimiento como activos para el desarrollo de las empresas hace que la llamada economía del conocimiento se caracterice por la necesidad del aprendizaje continuado y el desarrollo de competencias asociadas al uso efectivo de la información. (OECD, 2003). La OECD, en sus estudios sobre las competencias básicas para cualquier ciudadano (estudiadas en el proyecto $\mathrm{DeSeCo}$ ), cita entre éstas la alfabetización informacional. (Cuevas, 2005).

-El modelo educativo del Espacio Europeo de Educación Superior basado en las competencias destaca la relevancia de las informativas. El proyecto Tuning Educational Structures in Europe establece que las competencias genéricas o transversables son vitales en una sociedad cambiante y aquí incluye a la gestión de información. (Tuning, 2002).

-Así también el informe Developing lifelong learners through undergraduate education (Candy, 1994) cita a la competencia informacional como una de las competencias que permite que un individuo se pueda adaptar a los diferentes ambientes del desarrollo de su profesión.

-La Guía de la Agència per a la Qualitat del Sistema Universitari de Catalunya, cuando trata las competencias transversales o genéricas (...) plantea que se pueden clasificar en las categorías siguientes: gestión de tiempo y recursos, interpersonal, gestión de la información, personal, instrumental. (Agència per a la Qualitat del Sistema Universitari de Catalunya, 2006).

-Se incluye la alfabetización en Información dentro de la Década de la Alfabetización de las Naciones Unidas.

-La alfabetización informacional forma parte del programa de la UNESCO "Educación para todos". (UNESCO, 2005).

-En las dos últimas Cumbres Mundiales sobre la Sociedad de la Información se habla de una agenda de la solidaridad digital, en el que la biblioteca constituye un servicio público para el acceso libre y equitativo a la información, y como instrumento de mejora de la alfabetización en Tecnologías de Información y Comunicación y de la conectividad. (Cumbre Mundial para la Sociedad de la Información, 2005).

Se han desarrollado diversas iniciativas en este tema; dentro de las que se pueden mencionar diferentes: 
-Proyectos: European Network for Information Literacy (ENIL)

-Grupos de investigación: INFOSFERA de España que presenta diferentes proyectos e-learning relacionados con la alfabetización informacional y las competencias informacionales, entre otras líneas de actuación.

-Declaraciones y manifiestos:

La Declaración de Copenhague, en la que se debate el papel clave y las funciones de las bibliotecas públicas en las emergentes sociedades de la información, destacando su responsabilidad en la alfabetización y el aprendizaje permanente. (Declaración de Copenhague, 1999).

El Manifiesto de Oeiras, en el que se establecen unos objetivos y un plan de acción para mejorar el papel de las bibliotecas públicas, museos y archivos. (Manifiesto de Oeiras, 2003).

La Declaración de Praga, resultado de la Reunión de Expertos sobre Alfabetización Informacional, organizada por la U.S. National Comission on Library and Information Science y el National Forum on Information Literacy, con el lema "Hacia una sociedad alfabetizada en información”. (Declaración de Praga, 2003).

La Declaración de Alejandría, proclama que "la alfabetización informacional y el aprendizaje a lo largo de la vida son los faros de la sociedad de la información". (Declaración de Alejandría, 2005).

La Declaración de Toledo, surgida de un seminario de trabajo organizado con el lema "Biblioteca, aprendizaje y ciudadanía: la ALFIN" que reunió tanto a profesionales del mundo bibliotecario como del mundo educativo. (Declaración de Toledo, 2006).

-Eventos ${ }^{4}$ : Librarians' Information Literacy Annual Conference (LILAC); Encuentros de Desarrollo de Habilidades de Información (DHI).

Dentro de diferentes eventos, este tema se ha incluido de forma importante, como por ejemplo: Congreso Mundial de Bibliotecas e Información; Congreso Internacional de Información que se celebra en Cuba (INFO); Congreso Internacional de Bibliotecas Escolares en Lisboa (IASL); Conferencias anuales de LOEX (Library Orientation Exchange); Jornadas de Bibliotecas Escolares de la Fundación Germán Sánchez Ruipérez; Seminario "Nuevos desafíos para las Bibliotecas Universitarias"; Jornadas Españolas de Documentación FESABID; Jornadas REBIUN; Jornadas Bibliotecarias de Andalucía; Encuentros Internacionales sobre Sistemas de Información y Documentación (IBERSID);

4 Estos y otros eventos y recursos se encuentran en la página www.infolitglobal.info.
Jornadas de Gestión de Información de la Sociedad Española de Documentación e Información cienífica (SEDIC), Jornadas mexicanas de Biblioteconomía; Reunión de Bibliotecarios de la Península de Yucatán.

-Dentro de las revistas que desarrollan el tema se encuentran: Journal of Information Literacy, Information Research, Library Instruction Round Table News, Communications in information literacy, Library Journal, Anales de Documentación, Boletín de la Asociación Andaluza de Bibliotecarios, BiD: textos universitaris de Biblioteconomia i Documentació; Pinakes: Revista de las Bibliotecas Escolares de Extremadura, Revista Bibliotecas, School Library Media Quarterly, Reference and User services Quarterly, Bulletin of the ASIS, Library and Information Science Research, Ciencia da Informacao, American Libraries, Education Technology Journal, Journal of Information Science, Annual Review of Information Science and Technology, Reference Services Review, Science and Technology Libraries.

-Recursos en línea: Directorio de Recursos para el Desarrollo de Competencias informacionales (www. infolitglobal.info); Directory of Online Resources for Information Literacy (bulldogs.tlu.edu/mdibble/doril/); el Foro ALFINRED (www.alfinred.org); National Forum on Information Literacy (www.infolit.org); la lista de distribución Alfincat (alfincat@yahoogroups.com); el blog de Sheila Webber (information-literacy.blogspot.com); el blog Information Literacy in Canada (blog.uwinnipeg.ca/ilig/); el blog FORMIST de Chevillote (blogformist.enssib.fr); el portal ALFIN-EEES dirigido por María Pinto para la Educación Superior (www.mariapinto.es/alfineees); el portal Formation a la maitrise de l'information de Bernhard seguido por Lerouxe (www.ebsi.umontreal.ca/formanet/); el portal de habilidades informacionales (bivir.uacj.mx/dhi).

\section{CONSIDERACIONES FINALES}

Se puede concluir, a partir del estudio de las definiciones y tipologías de competencias, que éstas desde la perspectiva informacional, se hacen cada vez más transversales en función de los diferentes escenarios existentes; y que aunque aún existan determinadas controversias terminológicas pueden plantearse como competencias imprescindibles para la alfabetización en información.

Las disímiles iniciativas presentes imponen la gestión de las competencias informacionales - identificación, normalización, evaluación, formación y certificación - como componente eminente de cualquier entorno en la sociedad de hoy.

Artigo submetido em 26/09/2007 e aceito para publicação em 22/08/2008. 


\section{BIBLIOGRAFIA CITADA}

AFNOR. Norme française NF X 50-750: formation professionnelle: terminologie. París: ICS, 1996. Disponível em: < http://www.cinterfor. org.uy/public/spani sh/region/ampro/ cinterfor/temas/complab/doc/ otros/sel_efe/index.htm>. Acesso em: jan. 2007.

AGÈNCIA PER A LA QUALITAT DEL SISTEMA UNIVERSITARI DE CATALUNYA. Informe intern del CAI Servei de Biblioteques $i$ Documentació. 2006. Disponível em: < http://bibliotecnica.upc.edu/ avaluacioAqU2006/arxius/informe_intern_cai.pdf $>$. Acesso em: ago. 2006.

AGUDELO, S. El diagnóstico de competencias. Montevideo: CINTERFOR, 1998. Disponível em: < http://www.cinterfor.org.uy/ public/spanish/region/ampro/cinterfor/ publ/boletin/149/pdf/fanap. pdf >. Acesso em: nov. 2006.

ALA. Presidential commission on information literacy. 1989. Disponível em: <http:/www.ala.org/ala/acrl/acrlpubs/whitepapers/presidential. cfm l>. Acesso em: jan. 2007.

ALGO para todos: las bibliotecas públicas y la sociedad de la información. 1999. Disponível em: < http://travesia.mcu.es/ documentos/copenhague.pdf $>$. Acesso em: jan. 2007.

ANGULO, N. Normas de competencia en información. BiD: textos universitaris de biblioteconomia i documentació, n. 11, 2003. Disponível em: <http://www2.ub.es/bid/ consulta_articulos. php?fichero=11 langul2.htm $>$. Acesso em: 2007.

personal.

. Terminología. [S. L.: s. n.], 2007. Comunicación

ANSORENA, A. 15 casos para la selección de personal con éxito. Barcelona: Paidos, 1996. Disponível em: < http://www.cinterfor.org. uy/public/spanish/region/ampro/cinterfor/ temas/complab/doc/otros/ sel_efe/index.htm >. Acesso em: jan. 2007.

ANUIES. La educación superior en el siglo XXI: líneas estratégicas de desarrollo: una propuesta de la ANUIES. México, 2000. Disponível em: <http://www.anuies.mx> . Acesso em: jan. 2007.

BARRY, C. A. Information skills for an electronic world: training doctoral research students. Journal of Information Science, v. 23, n. 3, p. 225-238, 1997.

BAWDEN, D. Revisión de los conceptos de alfabetización informacional y alfabetización digital. Anales de Documentación, n. 5, p. 361-408, 2002. Disponível em: < http://www. um.es/fccd/anales/ad05/ad0521. pdf>. Acesso em: jan. 2007.

BEHRENS, S. J. A conceptual analysis and historical overview of information literacy. College and Research Libraries, v. 55, n. 4, p. 309322. Disponível em: < http://bbf. enssib.fr/sdx/BBF/frontoffice/2005/02/ document.xsp?id=bbf-2005-02-0042-007/2005/02/fam-dossier/dossier \&statutMaitre $=$ non\&statutFils=non $>$. Acesso em: jan. 2007.

BENITO, F. Nuevas necesidades, nuevas habilidades. Fundamentos de la alfabetización en información. In: GÓMEZ, J. A. (Coord.). Estrategias y modelos para enseñar a usar la información: guía para docentes, bibliotecarios y archiveros. Murcia: KR, 2000. p.11-75. Disponível em: < http://eprints.rclis.org/archive/00004672/02/EMPEUIcap1.pdf> . Acesso em: oct. 2006.
. Cuestiones previas a un proyecto ALFIN. In:

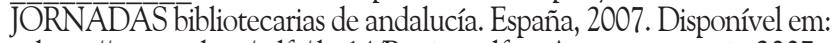
$<$ http://www.aab.es/pdfs/jba14/Benito.pdf > . Acesso em: maio 2007.

. ¿Qué es alfabetización informacional?. Pinakes: revista de las bibliotecas escolares de extremadura, año 1, n. 3, 2007b. Disponível em: <http://pinakes.educarex.es/ numero3/articulo5.htm $>$. Acesso em: maio 2007.

BIBLIOTECAS por el aprendizaje permanente. 2006. Disponível em: $<$ http://travesia.mcu.es/S_ALFIN/ficheros/Declaracion_Toledo.pdf $>$. Acesso em: jan. 2007.

BJONER, S. N. The information literacy curriculum: a working model. Latul Quarterly, 1991, v. 5, n. 2, p. 151-60

BOYATZIS, W. The competent manager: a model for effective performance. [S.l: s. n.], 1982.

BREIVIK, P. S. Putting libraries back in the information society. American Libraries, v. 6, n. 1, p. 723, 1985.

BRINDLEY, L. A world of contrasts: information literacy in the digital world. In: LILAC . Inglaterra: University of Leeds, 2006. Disponível em: < http://www.cilip.org.uk/NR/ rdonlyres/4B47053F-F385-4436902C-A1AE6CFE570F/0/brindley.ppt>. Acesso em: jan. 2007.

BROSE, F. K. Information competency and community college libraries: california moves toward a graduation requirement. Community and Junior College Libraries, v. 11, n. 1, p. 37-44, 2002.

BRUCE, C. Seven faces of information literacy in higher education. Queensland: University of Technology, 1997. Disponível em: <http:// sky.fit.qut.edu.au/\%7Ebruce/ inflit/faces/faces1.htm>. Aceso em: mar. 2007.

BUNK, G. P. La transmisión de las competencias en la formación y perfeccionamiento profesionales en la RFA. Revista CEDEFOP, n. 1 . p. 16, 1994.

BURCHINAL, LG. The communication revolution: America's third century challenge in the future of organising knowledge. Texas: A\&M University Library, 1976. Disponível em: < http://www.um.es/fccd/ anales/ad05/ad0521.pdf>. Acesso em: jan. 2007.

BYRNE, A. La alfabetización informacional desde una perspectiva global: el desastre agudiza nuestras mentes. Anales de Documentación, n. 8, p. 7-20, 2005. Disponível em: < http://www.um.es/fccd/anales/ ad08/ad0801.pdf>. Acesso em: mar. 2006.

CANDY, P. C; CREBERT G.; O'LEARY, J. Developing lifelong learners through undergraduate education. In: NATIONAL board of employment, education and training. Canberra: australian government publishing service, 1994. Report Núm. 28

. Running amock with a chainsaw: a personal perspective on Information Literacy (in three acts). In: LILAC. Inglaterra: University of Leeds, 2006. Disponível em: < http://www.cilip.org.uk/ NR/rdonlyres/E59CDDA1-COCB-42D6-86A4-79DF82CA90E4/0/ Candy.ppt>. Acesso em: jan. 2007.

CARDONA, P.; CHINCHILLA, M. N. Evaluación y desarrollo de las competencias directivas. Harvard Deusto Business Review, n. 89, p. 10-27, 1999. Disponível em: < http://kino.iteso.mx/ luisg/ 
EVALUACION\%20Y\%20DESARROLLO\%20DE\%20LAS\%20 COMPETENCIAS\%20DIRECTIVAS.doc>. Acesso em: set. 2006.

CEJAS, E.; PÉREZ, J. Un concepto muy controvertido: competencias laborales. Disponível em: <http://www.gestiopolis.com/recursos2/ documentos/fulldocs/rrhh/controcomplab. htm>. Acesso em: abr. 2006.

CHEVILLOTTE, S. Bibliothèques et information literacy: un état de l'art. Bulletin des Bibliothèques de France, v. 50, n. 2, p. 42-48, 2005. Disponível em: < http://bbf.enssib.fr/ sdx/BBF/frontoffice/2005/02/ document.xsp?id=bbf-2005-02-0042-007/2005/02/fam-dossier/dossi er\&statutMaitre $=$ non\&statutFils=non $>$. Acesso em: jan. 2007.

CONSEJO FEDERAL DE CULTURA Y EDUCACIÓN DE ARGENTINA. 2000. Disponível em: < http://www.cinterfor.org.uy/ public/spanish/region/ampro/cinterfor/publ/man_ops/index.htm >. Acesso em: maio 2006.

CÓRDOBA, S. Terminología. [S.1: s. n.], 2007. Comunicación personal.

CORNELLA, A. La educación en la sociedad del conocimiento.1999. Disponível em: < http://istserv.rediris.es/cgi-bin/wa?A2 = ind9904b\& $\mathrm{L}=$ iwetel $\& D=1 \& \mathrm{P}=5611>$. Acesso em: jan. 2007.

. Infonario: el diccionario de infonomía: español-inglés, english-spanish. Barcelona: Zero Factory, 2001. Disponível em: $<$ http://www.infonomia.com/blog/perm.php?id=2260>. Acesso em: jan. 2007.

CORTÉS, J. Competencias informativas y comunidades de aprendizaje: nuevos escenarios para los programas de formación de usuarios. In: REUNIÓN DE BIBLIOTECARIOS DE LA PENÍNSULA DE YUCATÁN, 10., 2003, Mérida. Anales electrónicos... Mérida, 2003. Disponível em: < http://bivir.uacj.mx/dhi/PublicacionesUACJ/Docs/ Ponencias/PDF/Ponmerida03.pdf > . Acesso em: jan. 2007.

COWLING, A.; JAMES P. La esencia de la administración de personal y las relaciones industriales. México: Prentice Hall Hispanoamericana, 1997. Disponível em: <http://www.cinterfor.org.uy/public/spanish/ region/ampro/cinterfor/publ/boletin/152/pdf/ir_va.pdf $>$. Acesso em: jan. 2006.

CRUZ, P. K. La gestión por competencias: una nueva herramienta en la planificación estratégica del recursos humano. 2001. Trabalho de conclusão de curso (Graduação em Administração de Empresas)Universidad de Antofagasta, Chile, 2001.

CUESTA, A. Gestión por competencias. Havana: Editorial Pueblo y Educación, 2001.

. Gestión del conocimiento: análisis y proyección de los recursos humanos. La Habana: Editorial Academia, 2002.

CUEVAS, A; VIVES, J. La competencia lectora en el estudio PISA: un análisis desde la alfabetización en información. Anales de Documentación, n. 8. 2005. Disponível em: < http://www.um.es/fccd/ anales/ad08/ad0804.pdf>. Acesso em: mar. 2006.

CURZON, S. C. Information competence in the CSU. California: CSU, 1995. Disponível em: < http://www.calstate.edu/LS/Archive/info comp_report.shtml>. Acesso em: jan. 2007.

DECLARACIÓN de Alejandría acerca de la alfabetización informacional y el aprendizaje de por vida. 2005. Disponível em: $<$ http://www.ifla.org/III/wsis/BeaconInfSoc-es.html > . Acesso em: jan. 2007.
DODGE, L. Building academic skills and information competency through learning communities. Educational Technology \& Society, v. 6, n. 3, p. 72-78, 2003. Disponível em: <http://www.ifets.info/ journals/6_3/9.html >. Acesso em: mar. 2006.

DOLAN, J. No time to lose...one literacy for all. In: LILAC. Inglaterra: Manchester Metropolitan University, 2007. Disponível em: < http:// www.cilip.org.uk/NR/rdonlyres/ E1DDB032- DEA0-45AE-A4D528203A10A3DB/0/Dolan07.ppt>. Acesso em: maio 2007.

DUCCI, M. El enfoque de competençia laboral en la perspectiva internacional. 1997. In: FORMACIÓN basada en competencia laboral. Montevideo : Cinterfor/OIT, [s. d.]. Disponível em: < http:// www.monografias.com/trabajos16/competencias/competencias. shtml\#Comentarios >. Acesso em: jan. 2007.

ENEBRAL, J. 2006: por la competencia en el manejo de la información. 2006. Disponível em: <http://www.degerencia.com/articulo/2006_por_ la_competencia_en_el_manejo_de_la_informacion $>$. Acesso em: jan. $2 \overline{00}$.

FATZER, J. B. Library literacy. RQ ., v. 26, n. 3, p. 313-314, 1987. Disponível em: < http://www.um.es/fccd/anales/ad05/ad0521.pdf >. Acesso em: jan. 2007.

FELIÚ, P.; RODRÍGUEZ, N. Manual del curso técnicas de entrevista y decisión de selección. Caracas: Psico Consult, 1994. Disponível em: $<$ http://www.cinterfor.org.uy/ public/spanish/region/ampro/cinterfor/ temas/complab/doc/otros/sel_efe/index.htm >. Acesso em: jan. 2007.

FJALLBRANT, N.; MALLEY, I. User education in libraries. 2. ed. London: Clive bingle, 1984. Disponível em: < http://informationr.net/ ir/8-4/paper159.html >. Acesso em: set. 2006.

GALLART, M. A; JACINTO, C. Competencias laborales: tema clave en la articulación educación-trabajo. Boletín Educación y Trabajo, año 6, n. 2, 1995. Disponível em: < http://www.campus-oei.org/oeivirt/fp/ cuad2a04.htm >. Acesso em: mar. 2006.

; NOVICK, M. Reestructuración industrial, capacitación y redes productivas. In: GALLART, M.A.; BERTONCELLO, R. Cuestiones actuales de la formación. Montevideo: Cinterfor, 1997. p. 63-92. Disponível em: <http://www.cinterfor. org.uy/public/spanish/ region/ampro/cinterfor/publ/papel/2/pdf/papeles2.pdf $>$. Acesso em: mar. 2006.

GARFIELD, E. 2001: an information society?. Essays of an Information Scientist, v. 6, p. 609-615, 1983. Disponível em: < http://www.garfield. library.upenn.edu/essays/ v6p609y1983.pdf > . Acesso em: mar. 2006.

$\mathrm{GOAD}, \mathrm{T}$. W. Information literacy and workplace performance. London: Quorum Books. 1998.

GOETSCH, L. A; KAUFMAN, P. T. Reading, writin, arithmetic, and information literacy: adding a basic skills component to a university's curriculum. Campus-Wide Information Systems, v. 15, n. 5, p. 158-63, 1998.

GÓMEZ, J. A. Estrategias y modelos para enseñar a usar la información: guía para docentes, bibliotecarios y archiveros. Murcia: KR, 2000. Disponível em: < http://eprints.rclis.org/archive/00004672/02/ EMPEUIcap1.pdf > . Acesso em: mar. 2006.

. Los problemas de las bibliotecas escolares de la Región de Murcia en un contexto de crisis del sistema educativo. Anales de Documentación, n. 5, p. 125-156, 2002. Disponível em: <http://www. um.es/fccd/anales/ad05/ad0507.pdf > . Acesso em: jan. 2007. 
- Alfabetización informacional: cuestiones básicas. 2005. Disponível em: $<$ http://www.thinkepi.net/repositorio/alfabetizacioninformacional-cuestiones-basicas/>. Acesso em: jan. 2007.

GONCZI, A; ATHANASOU, J. Instrumentación de la educación basada en competencias: perspectivas teóricas y prácticas en Australia. México: Limusa, 1996

GONZÁLEZ, V. La formación de la competencia profesional en la Universidad. Reflexiones y experiencias desde una perspectiva educativa. In: CONVENCIÓN INTERNACIONAL DE EDUCACIÓN SUPERIOR, 4., 2004, La Habana. Electronic Proceedings... Havana, 2004. Disponível em: <http://www.gestiopolis.com/recursos2/ documentos/ fulldocs/rrhh/controcomplab.htm>. Acesso em: 2008.

GRAMINIA, M. R. Gestión por competencias: una opción para hacer a las empresas más competitivas. 2000. Disponível em: <http://www. gestiopolis.com/recursos/documentos/ fulldocs/ger/gesporcompuch. $\mathrm{htm}>$. Acesso em: jan. 2006.

HAMEL, G.; PRAHALAD, C. K. Competing for the future. Boston: Harvard Business School Press, 1994.

HAMELINK, C. An alternative to news. Joumal of Communication, n. 26, p. 122, 1976. Disponível em: < http://www.um.es/fccd/anales/ad05/ ad0521.pdf>. Acesso em: jan. 2007.

HEPWORTH, M. Approaches to providing information literacy training in higher education: challenges for librarians. The New Review of Academic Librarianship, p. 21-34, 2000. Disponível em: <http:// informationr.net/ir/8-4/paper159.htm >. Acesso em: set. 2006.

HERNÁNDEZ, P. La formación de usuarios de la información en instituciones de educación superior. México: Cuib, 1998.

personal.

Terminología. [S. 1.: s. n.], 2007. Comunicación

HOOGHIEMSTRA, T. Gestión integrada de recursos humanos. In: LAS COMPETENCIAS: clave para una gestión integrada de los recursos humanos. [S. 1.]: Ediciones Deusto, 1996.

HORTON, F. W. Information literacy vs. computer literacy, Bulletin of the American Society for Information Science, v. 9, n. 4, p. 14-16, 1983.

IBARRA, A. Formación de los recursos humanos y competencia laboral. Boletin Cinterfor, n. 149, 2000. Disponível em: < http://www.cinterfor. org.uy/public/spanish/region/ampro/ cinterfor/publ/boletin/149/pdf/ ibarra.pdf>. Acesso em: set. 2006.

IHCD. Operational guidelines and advice for approved centers. Reino Unido: Bristol, 1998. Disponível em: < http://www.cinterfor.org.uy/ public/spanish/region/ampro/ cinterfor/publ/man_ops/index.htm $>$. Acesso em: maio 2006.

INEM. Metodología para la ordenación de la formación profesional ocupacional. Madrid: Subdirección general de gestión de formación ocupacional, 1995. Disponível em: < http://www.cinterfor.org.uy/ public/spanish/region/ampro/cinterfor/publ/man_ops/index.htm $>$. Acesso em: maio 2006.

IRIGOIN, M. E. En torno al concepto de competencias: programa de fortalecimiento de servicios de salud. Perú: [s. n.], 1996.

; VARGAS, F. Competencia laboral: manual de conceptos, métodos y aplicaciones en el sector salud. Montevideo: Cintefor, 2002. Disponível em: <http:// www.cinterfor.org.uy/public/spanish/region/ ampro/cinterfor/publ/man_ops/index.htm >. Acesso em: maio 2006.
. Competencia laboral: manual de conceptos, métodos y aplicaciones en el sector salud. Montevideo: Cintefor, 2002. Disponível em: < http://www.cinterfor.org.uy/public/spanish/region/ampro/ cinterfor/publ/man_ops/index.htm>. Acesso em: maio 2006.

KOCHANSKY, J. El sistema de competencias. In: TRAINING and development digest. Madrid. 1998.

KUHLTHAU, C. C. Information search process: a summary of research and implications for school library media programs. School Library Media Quarterly, v. 22, n. 1, p. 19-25, 1989. Disponível em: < http://www. ala.org/ala/aasl/aaslpubsandjournals/slmrb/ editorschoiceb/infopower/ selectkuhlthau2.cfm>. Acesso em: jan. 2007.

LASIDA, J. Competencias laborales: un instrumento para el diálogo y la negociación entre educación y trabajo. In: SEMINARIOORGANIZACIÓN Y GESTIÓN DE PROYECTOS DE REFORMA DE FORMACIÓN PROFESIONAL BASADA EN COMPETENCIAS, 1998, Montevideo. Anales electrónicos... Montevideo, 1998. Disponível em: < http://www.oei.org.co/ iberfop/ uruguay $1 . h$ tm > . Acesso em: ago. 2006.

LAU, J. Terminología. [S. 1.: s. n.], 2007. Comunicación personal.

LE BOTERF, G. De la compétence à la navigation professionnelle. París: Les Editions d'Organisation, 1997. Disponível em: < http://www.unige. ch/fapse/SSE/groups/life/livres/LeBoterf_R1997_A.html >. Acesso em: mar. 2006.

LENOX, M. F; WALTER, M. L. Information literacy: challenge for the future. International Journal of Information and Library Research, $\mathrm{n}$. 4, p. 1-18, 1992. Disponível em: <http://www.um.es/fccd/anales/ad05/ ad0521.pdf>. Acesso em: jan. 2007.

LEVY-LEBOYER, C. Gestión de las competencias: cómo analizarlas, cómo evaluarlas, cómo desarrollarlas. 1997 Madrid: Gestión, 2000. Disponível em: < http://www.gestiopolis. com/recursos/documentos/ fulldocs/rrhh/sobrecomp.htm >. Acesso em: abr. 2006.

MACKENZIE, J. The information literacy school community. The Education Technology Journal, v. 8, n. 1, 1998. Disponível em: < http:// eprints.rclis.org/archive/00004672/02/EMPEUIcap1.pdf > . Acesso em: out. 2006.

MANIFIESTO de Oeiras. 2003. Disponível em: < http://www. pulmanweb.org/documents/manifesto/manifesto-spanish-version. doc >. Acesso em: 2008.

MANUEL, K. Generis and discipline-specific information literacy competencies: the case of the sciences. Science and Technology Libraries, v. 24, n. 3-4, p. 279-308, 2004. Disponível em: < http://624. brianwinterman.com/manuel.pdf $>$. Acesso em: mar. 2006.

MCCLELLAND, D. C. Testing for competence rather than for intelligence. American Psychologist, v. 28, n. 1, p. 1-14, Jan. 1973.

MCCLURE, C. R. Network literacy: a role for libraries. Information Technology and Libraries, n. 13, p. 115-125, 1994.

MERTENS, L. Competencia laboral: sistemas, surgimiento y modelos. Montevideo: Cintefor, 1996. Disponível em: < http://www.cinterfor.org. uy/public/spanish/region/ampro/cinterfor/publ/mertens/pdf/mertens. pdf $>$. Acesso em: jan. 2007.

. De la calificación a la competencia Competencia Laboral., v. 6, n. 22, p.6-13, 2002. Disponível em: < http://www.cinterfor.org. uy/public/spanish/region/ampro/cinterfor/temas/complab/doc/otros/ mert04.htm>. Acesso em: jan. 2007. 
MONTERO, M.; ROCA, M. La formación en competencia informacional: el bibliotecario en el aula. In: CONGRESO UNIVERSITARIO DE INNOVACIÓN EDUCATIVA EN LAS ENSENANZAS TÉCNICAS, 2004, Barcelona. Anales electrónicos... Barcelona, 2004. Disponível em: <http://eprints.rclis.org/ archive/00002479/01/279.pdf> . Acesso em: jan. 2007.

MORALES, E. Latin America and the Caribbean. In: WORLD information report, 1997-98. p. 167-180. Disponível em: $<$ http:/144.16.65.194/hpg/envis/doc97html/enwir212. html > . Acesso em: maio 2007.

MOREIRA, J.A. Terminología. [S. 1.: s. n.], 2006. Comunicación personal.

MORENO, J. Modelo Integrado de gestión por competencias. Revista Capital Humano, n. 125. p. 12-15, 1999.

MUNTADA, M.; et. al. Competència en el maneig d'informació: punt d'arribada o punt de sortida de la formació d'usuaris?. 2003. Disponível em: <http://eprints.rclis.org/archive/00000524/01/Competencia.pdf> . Acesso em: jan. 2007.

MUÑOZ, J. Implantación de un sistema de selección por competencias. Training and Development Digest, n. 10, 1998.

NOVICK, M. et al. Nuevos puestos de trabajo y competencias laborales: un análisis cualitativo en el sector metalmecánico argentino. Montevideo: Cintefor, 1998. Disponível em: < http://www.cinterfor.org.uy/public/ spanish/region/ampro/cinterfor/publ/papel/6/pdf/novick.pdf> . Acesso em: nov. 2006

OECD. Deseco project. 2003. Disponível em: < http://www.portal-stat. admin.ch/deseco/index.htm>. Acesso em: jan. 2007.

OLSEN, J. K.; COONS, B. Cornell University's information literacy program. In: MENSCHING G. E; MENSCHING, T. B. (Ed.). Coping with information illiteracy: bibliographic instruction for the information age. Ann Arbor, MI: Pieran Press, 1989. p. 7-20. Disponível em: $<$ http://www.um.es/fccd/anales/ad05/ad0521.pdf > . Acesso em: jan. 2007.

ORTOLL, E. Competencia informacional en las ciencias de la salud. Propuesta de un modelo de formación. Tesis (Doctoral)- Universidad de Zaragoza, Zaragoza, 2003. Director: Isabel Ubieto.

OWENS, M. R. State covermnent and libraries. Library Joumal, v. 101, n. 1, p. 19-28, 1976. Disponível em: <http:/www.um.es/fccd/anales/ ad05/ad0521.pdf>. Acesso em: jan. 2007.

PASADAS, C. The international information literacy certificate: a global professional challenge?. In: CONGRESO MUNDIAL DE BIBLIOTECAS E INFORMACIÓN; CONGRESO GENERAL Y CONSEJO DE LA IFLA, 2003, Alemania. Anales electrónicos... Alemanha, 2003. Disponível em: < http://www.ifla.org/IV/ifla69/ papers/202e_trans-Pasadas_Urena.pdf > . Acesso em: jan. 2007.

. La alfabetización informacional (ALFIN) en la Enseñanza Superior. In: LA BIBLIOTECA en el entorno del espacio europeo de educación superior. [S. 1.]: Universidad de Verano de Adeje, 2004.

; ÁLVAREZ, F. J. Formación de los ciudadanos en competencias para el manejo de la información: una propuesta de actuación para Andalucía: resumen ejecutivo. In: JORNADAS BIBLIOTECARIAS DE ANDALUCIA, 2007, Málaga. Anales electrónicos... Málaga: Antequera, 2007. Disponível em: <http://www. aab.es/pdfs/jba14/PasadasAlvarez.pdf>. Acesso em: maio 2007.
PINTO, M. Alfabetización informativo-digital. 1991. Disponível em: $<$ http://www. mariapinto.es/e-coms/alfa_infor.htm $>$. Acesso em: jan. 2007.

; GARCÍA, J. La enseñanza-aprendizaje de las competencias genéricas en el Espacio Europeo de Educación Superior: el proyecto ALFINEES. 2006. Disponível em: < http://www.unizar.es/ eees/innovacion06/COMUNIC_PUBLI/BLOQUE_II/CAP_II_9. pdf >. Acesso em: jan. 2007.

PISSINATI, T. El proyecto como fuente, método y proceso pedagógico de desarrollo del protagonismo en el espacio-tiempo de la educación tecnológica. ENCUENTROSOBRE FORMACIÓN TECNOLÓGICA DE EUROPA Y AMÉRICA LATINA, 3., 2002, La Havana. Anales electrónicos... Havana, 2002. Disponível em: < http://www.gestiopolis. $\mathrm{com} /$ recursos2/documentos/fulldocs/rrhh/controcomplab.htm $>$. Acesso em: abr. 2006.

PLAN de acción. In: CUMBRE MUNDIAL PARA LA SOCIEDAD DE LA INFORMACIÓN, 2005, Túnez. Electronic proceedings...Túnez, 2005. Disponível em: <http://www.itu.int/wsis/docs2/tunis/off/6rev1es.pdf $>$. Acesso em: jan. 2007.

PONJUÁN, G. Gestión de información: dimensiones e implementación para el éxito organizacional. Argentina: Nuevo parhadigma, 2004.

RADER, H. B. Bibliographic instruction or information literacy. College and Research Libraries News, v. 51, n. 1, p. 18-20, 1991.

RAVITSKY, M. Metodología francesa: diseño de una acción de capacitación. In: ENCUENTRO, SOBRE FORMACIÓN TECNOLÓGICA DE EUROPA Y AMÉRICA LATINA, 3., 2002, La Habana. Anales... Havana, 2002. Disponível em: < http://www. gestiopolis.com/recursos2/documentos/fulldocs/rrhh/controcomplab. $\mathrm{htm}>$. Acesso em: abr. 2006.

REIS, O. Cualificación contra competencia: debate semántico, ievolución de conceptos o baza política?. Berlín: CEDEFOP, 1994. n. 2. Disponível em: < http://www.monografias. com/trabajos16/competencias/ competencias.shtml\#Comentarios>. Acesso em: jan. 2007.

ROGERS, R. Teaching information skills: a review of the research and its impacto n education. London: Bowker-Saur, 1994. Disponível em: $<$ http://informationr.net/ir/8-4/paper159.html >. Acesso em: set. 2006.

RUIZ, L. PAHO. Desarrollo de la educación en salud pública: desafíos para el Siglo XXI. In: CONFERENCIA DE LA ASOCIACIÓN LATINOAMERICANA Y DEL CARIBE DE EDUCACIÓN EN SALUD PÚBLICA - ALAESP, 2000, Cuba. Anales...Cuba, 2000.

SCANS. What work requires of schools. 1991. Disponível em: < http:// wdr.doleta.gov/ SCANS/whatwork/whatwork.pdf $>$. Acesso em: jan. 2007.

SHAPIRO, J. J; HUGHES, S. K. Information literacy as a liberal art: enlightenment proposals for a new curriculum. Educom Review, v. 31, n. 2, p. 31-5, 1996 .

SNAVELY, L; COOPER, N. Competing agendas in higher education: finding a place for information literacy. Reference and User Services Quarterly, v. 37, n. 1, p. 53-62, 1997. Disponível em: <http://www. um.es/fccd/anales/ad05/ad0521.pdf>. Acesso em: jan. 2007.

SONNTAG, G. Alfabetización informacional y el rol de la biblioteca. Bibliotecas, v. 25, n. 1, 2007. Disponível em: < http://www.una.ac.cr/ bibliotecologia/boletinbiblioteca/2007/ articulogabrielasonntag.pdf $>$. Acesso em: maio 2007. 
SPENCER, L. M.; SPENCER, S. M. Competence at work. New York: John Wiley and Sons, 1993. Disponível em: < http://www.cinterfor.org. uy/public/spanish/region/ampro/ cinterfor/temas/complab/doc/otros/ sel_efe/index.htm>. Acesso em: jan. 2007.

.; MCCLELLAND, D. Competency assessment methods: history and state of the art. [S. 1.]: Hay/Mc.Ver Research Press, 1994. Disponível em: < http://www.cinterfor. org.uy/public/spanish/region/ ampro/cinterfor/publ/man_cl/index.htm >. Acesso em: nov. 2006.

SPITZER, K. L; EISENBERG, M. B; LOWE, C. A. Information literacy: essential skills for the information age. Syracuse: ERIC Clearinghouse on Information Literacy, 1998.

TALLER SOBRE COMPETENCIAS LABORALES, 2002. Anales electrónicos.... Disponível em: < http://www.gestiopolis.com/recursos2/ documentos/fulldocs/rrhh/ controcomplab.htm $>$. Acesso em: abr. 2006.

TAYLOR, R. S. Reminiscing about the future. Library Journal, n. 104, p. 1871-5, 1979.

. Value-added processes in information systems. [S. 1.]: Ablex Publishing Corp., 1986. Disponível em: <http://www.um.es/fccd/ anales/ad05/ad0521.pdf>. Acesso em: jan. 2007.

TEDESCO, J. C. La política educativa en la sociedad del conocimiento y de la información. El desafío de educar, n. 15, 2000. Disponível em: $<$ http://orozco.cc/Educa/Docus/ 04tedesco5.htm > . Acesso em: jan. 2007.

TEJADA, J. Acerca de las competencias profesionales. Revista Herramientas, n. 57, 1999. Disponível em: < http://dewey.uab.es/ pmarques/dioe/competencias.pdf $>$. Acesso em: nov. 2006.

TREMBLAY, G. Pedagogía colegial. 1994. Disponível em: < http://www. gestiopolis.com/recursos2/documentos/fulldocs/rrhh/controcomplab. htm >. Acesso em: abr. 2006.

TUCKETT, H. W. Computer literacy, information literacy and the role of the instruction librarian. In: MENSCHING G. E; MENSCHING, T. B. (Ed.). Coping with information illiteracy: bibliographic instruction for the information age. Ann Arbor, MI: Pieran Press, 1989. p 21-3. Disponível em: < http://www.um.es/fccd/anales/ad05/ad0521.pdf>. Acesso em: jan. 2007.

TUNING educational structures in Europe. 2002. Disponível em: $<$ http://www.relint. deusto.es/TUNINGProject/spanish/doc_fase1/ Tuning\%20Educational.pdf> . Acesso em: jan. 2007.

UNESCO. Information literacy. In: . Information for all programme. 2005. Disponível em: < http://portal.unesco.org/ci/ en/ev.php-URL ID = 21293\&URL DO=DO_ TOPIC\&URL SECTION=201.html $>$. Acesso em: jan. 2007.

UREÑA, Cristóbal Pasadas. Hacia una sociedad alfabetizada en información. 2003 Disponível em: <http://melangeinfo.com/Doc/ Declaraci_Praga_castellano.pdf $>$. Acesso em: jan. 2007.

VARGAS, F. De las virtudes laborales a las competencias claves: un nuevo concepto para antiguas demandas. Boletín Cinterfor, n. 149, 2000. Disponível em: < http://www.cinterfor. org.uy/public/spanish/ region/ampro/cinterfor/publ/boletin/149/pdf/__varg.pdf > . Acesso em: 2008.
. 40 preguntas sobre competencia laboral. Montevideo: Cinterfor, 2004. Disponível em: < http://www.cinterfor.org.uy/public/ spanish/region/ampro/cinterfor/publ/papel/13/index.htm>. Acesso em: nov. 2006.

VIRKUS, S. Information literacy in Europe: a literatura review. Information Research, v. 8, n. 4, 2003. Disponível em: <http:// informationr.net/ir/8-4/paper159.html > . Acesso em: set. 2006.

WATSON, M. The need to embrace information competency across the spectrum. Information World Review, n. 196, p. 10, 2003.

WEBBER, S. An international information literacy certificate: opportunity or dead-end?. In: CONGRESO MUNDIAL DE BIBLIOTECAS E INFORMACIÓN; CONGRESO GENERAL Y CONSEJO DE LA IFLA, 2003, Alemania. Anales electrônicos... Alemanha, 2003. Disponível em: < http://www.ifla.org/IV/ifla69/ papers/199e-Webber.pdf> . Acesso em: jan. 2007.

WEINER, S. T.; HONORA F. N. Information competencies: a strategic approach. In: AMERICAN SOCIETY FOR ENGINEERING ANNUAL CONFERENCE AND EXPOSITION, 2001. Electronic proceedings... 2001. Disponível em: < http://www.asee.org/acPapers/ code/getPaper.cfm?paperID=3385\&pdf=00510 2001.PDF $>$. Acesso em: jan. 2007.

WORDRUFFE, C. What is meant by a competency? Leadership and Organisation Development Journal, v. 14, p. 29-36, 1993. Disponível em: $<$ http://www.gestiopolis. com/recursos2/documentos/fulldocs/rrhh/ controcomplab.htm >. Acesso em: abr. 2006.

WURMAN, R. S. Information anxiety. New York: Doubleday, 1989.

ZARIFIAN, P. A gestao da e pela competência. In: SEMINARIO INTERNACIONAL EDUCAÇÃO, TRABALHO E TRANSFERENCIA DE TECNOLOGIA, 1996, Rio de Janeiro. Anais eletrônicos... Rio de Janeiro: CIET, 1996. Disponível em: < http:// www.cinterfor.org.uy/ public/spanish/region/ampro/cinterfor/publ/ papel/6/pdf/novick.pdf > . Acesso em: nov. 2006.

. El modelo de la competencia y sus consecuencias sobre el trabajo y los oficios productivos. Montevideo: Cinterfor, 1999. Disponível em: < http://www.cinterfor.org.uy/public/spanish/region/ampro/ cinterfor/publ/papel/8/pdf/papel8.pdf> . Acesso em: nov. 2006.

ZAYAS, P. M. Las competencias como principal manifestación de la interrelación hombre - trabajo. 2003. Disponível em: <http:// www.monografias.com/trabajos16/ competencias/competencias. shtml\#Comentarios>. Acesso em: jan. 2007.

ZURKOWSKI, P. G. The information service environment relationships and priorities. Washington, DC: National Commission on Libraries and Information Science, 1974. Disponível em: < http://www.um.es/fccd/ anales/ad05/ad0507.pdf>. Acesso em: jan. 2007. 